\title{
Review: immunosuppression for the lung transplant patient
}

\author{
Sakhee Kotecha, Steven Ivulich, Gregory Snell \\ Lung Transplant Service, Alfred Hospital and Monash University, Melbourne, Australia \\ Contributions: (I) Conception and design: All authors; (II) Administrative support: All authors; (III) Provision of study materials or patients: None; \\ (IV) Collection and assembly of data: All authors; (V) Data analysis and interpretation: All authors; (VI) Manuscript writing: All authors; (VII) Final \\ approval of manuscript: All authors. \\ Correspondence to: Dr. Sakhee Kotecha. Lung Transplant Service, Alfred Hospital, Commercial Rd, Melbourne, 3004, Australia. \\ Email: s.kotecha@alfred.org.au.
}

\begin{abstract}
Lung transplantation (LTx) has evolved significantly since its inception and the improvement in LTx outcomes over the last three decades has predominantly been driven by advances in immunosuppression management. Despite the lack of new classes of immunosuppression medications, immunosuppressive strategies have evolved significantly from a universal method to a more targeted approach, reflecting a greater understanding of the need for individualized therapy and careful consideration of all factors that are influenced by immunosuppression choice. This has become increasingly important as the demographics of lung transplant recipients have changed over time, with older and more medically complex candidates being accepted and undergoing LTx. Furthermore, improved survival post lung transplant has translated into more immunosuppression related comorbidities long-term, predominantly chronic kidney disease (CKD) and malignancy, which has required further nuanced management approaches. This review provides an update on current traditional lung transplant immunosuppression strategies, with modifications based on preexisting recipient factors and comorbidities, peri-operative challenges and long term complications, balanced against the perpetual challenge of chronic lung allograft dysfunction (CLAD). As we continue to explore and understand the complexity of LTx immunology and the interplay of different factors, immunosuppression strategies will require ongoing critical evaluation and personalization in order to continue to improve lung transplant outcomes.
\end{abstract}

Keywords: Immunosuppression; lung transplant

Submitted Jun 23, 2020. Accepted for publication Feb 16, 2021.

doi: $10.21037 /$ jtd-2021-11

View this article at: http://dx.doi.org/10.21037/jtd-2021-11

\section{Introduction}

While lung transplantation (LTx) starts with extensive surgery in a patient with end-stage lung disease, it is actually the balance of intrinsic host immunity and extrinsic added immunosuppressive strategies that will most likely ultimately determine the long-term success of this therapy. The general management of immune deficient patients and the use of immunosuppressive agents has some commonality across different types of solid organ transplantation (SOT) — but the transplanted lung has some unique properties that require extra consideration. Particular issues for the lung allograft include: exposure to the external environment, the absence of lymphatic, bronchial arterial reconnection or cilial innervation, being the primary site of several infections problematic in all immune deficient patients [e.g., cytomegalovirus (CMV), pneumocystis] and being co-transplanted with whole lymph nodes of donor immune cells (1). Starting with the recipient, and moving through common early post-LTx scenarios and onto longer-term immunosuppression maintenance issues, the current review will consider the clinician and pharmacist's challenges and options.

\section{Modification of early immunosuppression}

In decades past, LTx immunosuppression strategies followed 
Table 1 The Alfred Hospital's current LTx 'Induction and initiation of Immunosuppression' protocol $(1,2)$

Induction

* Tacrolimus: $5 \mathrm{mg}$ orally if weight $>50 \mathrm{~kg}$, and $3 \mathrm{mg}$ po if weight $<50 \mathrm{~kg}$

Tacrolimus should not be given to patients on bosentan, azoles, orkambi or age $>55$ with borderline renal function

* Azathioprine: $2 \mathrm{mg} / \mathrm{kg}$ orally on acceptance of organs

Mycophenolate mofetil $500 \mathrm{mg}^{-1} \cdot \mathrm{gm}$ may be preferred in select patients (i.e., those who are (sensitized or have low TPMT level i.e., $<0.50$ ) as discussed with transplant physician

Intra-operative

* Methylprednisolone 500 mg intravenously on reperfusion of each lung

Early post-operative

* Methylprednisolone:

- $75 \mathrm{mg}$ (50 mg if weight $<50 \mathrm{~kg}$ ) intravenously every 8 hours for three doses followed by

- $1 \mathrm{mg} / \mathrm{kg}$ at 10.00 am daily, weaning by 5-10 mg every day until $20 \mathrm{mg} /$ day (Intravenous or oral as tolerated)

* Tacrolimus:

- Aim to commence within 12 hours of arrival in ICU (assuming adequate urine output and renal function). Initial dose should be delayed or lowered in patients:

- Taking bosentan, azoles or orkambi

- Renal impairment

- Day 0-1: >50 kg 0.5 mg intravenously twice daily; (<50 kg $0.3 \mathrm{mg})$ as a 4-hour infusion

- Day 2-4: Convert to oral administration; 10:1 conversion (i.e., $0.5 \mathrm{mg}$ IV tacrolimus is equivalent to 5 mg oral tacrolimus)

- Daily through levels and adjust, targeting a trough level of $10-12 \mathrm{mcg} / \mathrm{L}$

* Azathioprine:

- $1.5 \mathrm{mg} / \mathrm{kg} /$ day intravenously or orally daily

- If TPMT activity $<0.5$ then consider mycophenolate

- Sensitized patients to commence mycophenolate mofetil with target dose $1 \mathrm{gm}$ twice daily if $>50 \mathrm{~kg} ; 15 \mathrm{mg} / \mathrm{kg}$ if $<50 \mathrm{~kg}$

* Basiliximab:

- $20 \mathrm{mg}$ given intravenously days 0 and 4 in selected patients

Indications for use:

- Baseline renal impairment

- Complicated surgical procedure, shock state or poor urine output in ICU where renal injury is anticipated

- Pediatric patients (dose of $10 \mathrm{mg}$ to be used where $<35 \mathrm{~kg}$ )

LTx, lung transplantation.

the pathway of other more numerous SOT (particularly kidney transplantation), but given the above features there has been definite evolution to modify practice to enhance outcomes $(1,2)$. So in 2020, while LTx immunosuppression typically starts with a combination of the calcineurin inhibitor (CNI) tacrolimus, an anti-proliferative agent (azathioprine or mycophenolate), corticosteroids and an IL-2 blocker (basiliximab) (Table 1), clinical situations or infection or rejection events very commonly require individualization and revision of the initial protocol 


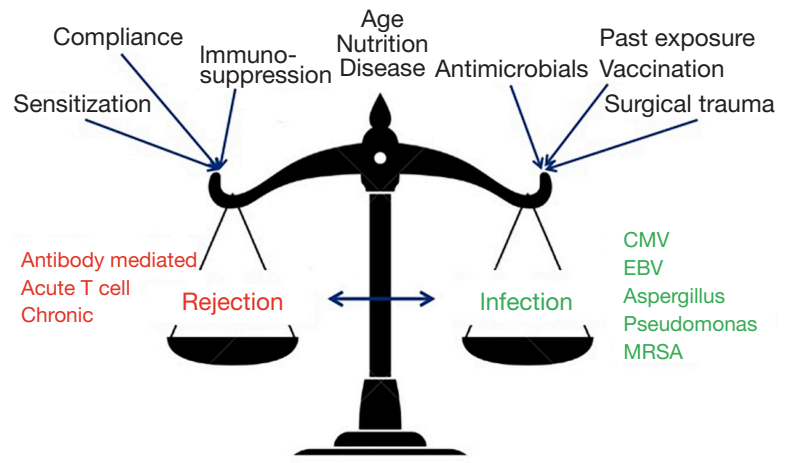

Figure 1 The concept of the net balance of immunosuppression in LTx. LTx, lung transplantation.

(Figures 1,2) (1-4).

\section{Recipient bost factors and immunosuppression}

\section{Intrinsic immune deficiency, advancing age}

A small cohort of patients receiving a transplant may be intrinsically immunosuppressed from the pre-existing indication for transplant. The risk of acute rejection in these patients may be lower and immunosuppression with induction agents may not be required. Following a "one size fits all approach" would put the patient at higher risk of sepsis in the perioperative period if the immunosuppression is not tailored to the specific infection risk of the patient.

Lung transplant recipients transplanted for bronchiectasis have a higher infection burden due to being intrinsically immunosuppressed. A more cautious approach with immunosuppression in the perioperative period may be required as bronchiectasis transplant recipients are predicted to suffer from higher rates of infection (5). Common variable immune-deficiency (CVID) is not a common indication for lung transplant with variable outcomes reported (6). CVID can predispose patients to recurrent infections, leading to the development of bronchiectasis, irreversible lung damage and eventual need for LTx (7). Immunoglobulin (IVIG) plays an important role in the management of immunosuppression post LTx. IVIG has immune-modulatory effects as well as an antiinfective role and can be utilized to minimise the higher risks of infection (8). Mycophenolate is known to have effects on IgG production and is associated with lower IgG levels after transplant due to its strong anti-proliferative effects on B-lymphocyte function $(7,9)$.

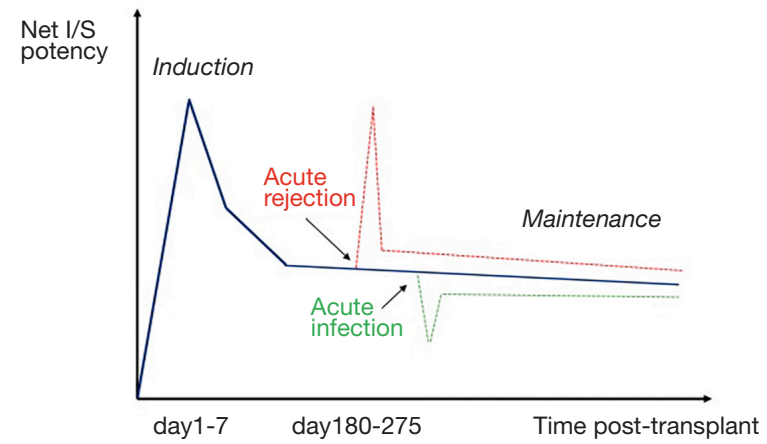

Figure 2 Current targeting strategies for LTx immunosuppression. LTx, lung transplantation.

Immunosenescence may predispose elderly patients to the risks of over-immunosuppression and theoretically less immunosuppression may be needed compared to younger patients. Elderly patients have lower rates of acute rejection and higher rates of bacterial infections and malignancies (10). In the perioperative period, the use of induction agents in older patients ( $>65$ years) may not be appropriate in recipients who are at higher risk of infection, such as those with CMV mismatch or at higher risk of sepsis.

Longer life expectancies and modern antiretroviral therapies have ensured that HIV-positive recipients are now considered for LTx with favourable outcomes reported. Data suggests that HIV recipients should receive induction immunosuppression due to concerns with viral suppression and increased infection risk. However, the acute rejection rate is higher for HIV infected transplants in other SOTs suggesting an excessively cautious use of immunosuppression. Ongoing larger studies are investigating the higher rejection rates in other SOTs and the optimum approach to immunosuppression in the HIVpositive recipients (11).

\section{Acquired immune deficiency}

Many potential LTx recipients come to transplant already affected by immunosuppressant medications. This could be a modest dose of prednisolone for refractory airways disease, pirfenidone for pulmonary fibrosis, a mammalian target of rapamaycin (mTOR) inhibitor (e.g., sirolimus) for lymphangioleiomyomatosis—right through to complex multidrug regimens or monoclonal antibodies that have been indicated for a prior transplant or autoimmune lung diseases. These agents often require modification of LTx induction therapy to avoid impacting early transplant 
Table 2 Immunosuppressive or immunomodulatory therapies used in potential LTx recipients that require consideration of modifying immunosuppression

\begin{tabular}{|c|c|c|c|}
\hline Medication/therapy & Indication & Specific effect on allograft/recipient & $\begin{array}{l}\text { Induction immunosuppression } \\
\text { modification }\end{array}$ \\
\hline Corticosteroids & $\begin{array}{l}\text { - Obstructive airways disease } \\
\text { - ILD }\end{array}$ & Poor wound healing & Decrease induction steroid dose \\
\hline $\begin{array}{l}\text { Tacrolimus, } \\
\text { Cyclosporine }\end{array}$ & Prior transplant & Poor renal function & Delay re-start of drug \\
\hline & - Prior transplant & - Non-specific & Anti-proliferative or steroid dose \\
\hline Pirfenidone, Nintedanib & ILD & Poor wound healing & Decrease induction steroid dose \\
\hline Rituximab & Autoimmune ILD & $\begin{array}{l}\text { - Interferes with donor/recipient cross match } \\
\text { - Lasts } 9 \text { months }\end{array}$ & No change \\
\hline $\begin{array}{l}\text { Intravenous gamma } \\
\text { globulin }\end{array}$ & $\begin{array}{l}\text { - Prior immune deficiency } \\
\text { - Highly sensitized }\end{array}$ & $\begin{array}{l}\text { - Interferes with serology testing } \\
\text { - Anti-rejection/anti infective }\end{array}$ & $\begin{array}{l}\text { Lost with haemorrhage- so } \\
\text { replace early }\end{array}$ \\
\hline
\end{tabular}

LTx, lung transplantation; ILD, interstitial lung disease.

outcomes (2), see Table 2.

\section{Specific challenging recipient infective or commensal organisms}

There are several scenarios where the risk of uncontrolled periLTx infection is known or suspected to be very challenging. Clear-cut examples include the isolation of Burkbolderia Cepacia Complex (12) or Mycobacterium Abscessus (13). The potential for complications mandates a specific reduced immunosuppression plan that dovetails existing co-morbidities (e.g., gastro-intestinal symptoms, renal impairment), anti-infective agent side-effects and antirejection immunosuppressant mode of action and sideeffects. Delayed introduction of the CNI, the routine use of basiliximab and a reduced dose of anti-proliferative agents and steroids is a good starting point (2). Avoidance of potent or long-acting agents such as high-dose pulsed steroids, anti-thymocyte globulin or rituximab is critical. Photophoresis, although an expensive and uncommon therapy, may provide an efficacious and safe alternative mode of immunomodulation (14).

\section{Specific recipient co-morbidities Neurological}

Patients with pre-existing neurological conditions may require special consideration leading into LTx. Elderly patients at risk of stroke are likely to require additional pre-transplantation workup. The incidence of stroke, although lower than other solid organ transplants, is $2 \%$ to $3 \%$ after lung transplant (15).

Patients with epilepsy need special consideration in the perioperative period. Seizures with CNIs are often generalized and can occur even when serum levels are therapeutic. Many treatments for seizures also have the potential to interact with immunosuppressant regimens due to their hepatic metabolism and cytochrome interactions. Levetiracetam is the drug of choice for post-transplant due to the minimal drug interactions (15).

\section{Gastrointestinal}

A small cohort of lung transplants recipients may be prescribed immunosuppressants for a range of autoimmune and inflammatory diseases such as rheumatoid arthritis, ulcerative colitis and Crohn's disease and may be significantly immunosuppressed leading into LTx. Treatments may vary from mild immunosuppression such as azathioprine to more potent cytokine modulators such as infliximab or adalimumab. Azathioprine, utilized in many regimes for inflammatory bowel disease, is also incorporated into standard immunosuppressant regimens. However, other disease modifying agents such as sulfasalazine or 
mercaptopurine are usually ceased post-LTx.

\section{Hematological}

Lung transplant is a feasible option for patients with severe pulmonary chronic graft versus host disease (GVHD). Patients with GVHD are likely to have had a complicated course coming into LTx with IVIG replacement complicating serological testing, risk of opportunistic infections post allogeneic hematopoietic stem cell transplant (HSCT) and deficiencies in bone marrow reserve after many years of receiving immunosuppression (16). Patients with GVHD are likely to benefit from less intense immunosuppressive regimens post LTx. A retrospective Japanese study demonstrated that postoperative complications within the first year after LTx can be minimized by using a lower dose of prednisolone (17).

Induction agents such as the T-cell depleting agents (anti-thymocyte globulin, alemtuzumab, basiliximab) or anti-metabolites can all cause varying degrees of leukopenia (18). Testing for thiopurine methyltransferase (TPMT) activity is required to ensure LTx recipients can adequately metabolize that 6-mercaptopurine. Those without adequate TPMT activity are at risk of leukopenia from azathioprine, the pro-drug of 6-mercatopurine. Reduced activity is seen in $10 \%$ of Caucasians with a smaller cohort completely absent of activity. In patients with low TPMT activity, an alternative antimetabolite such as mycophenolate would be given (19).

\section{Specific recipient drug interactions}

Patients receiving a lung transplant may be taking a medication that has the potential to destabilize immunosuppressant regimens if continued in the perioperative period. Many of these medications, such as the gene modifiers for cystic fibrosis (CF) and endothelin receptor antagonists for pulmonary hypertension are usually ceased once the transplant has taken place. However, their impact may linger for a few days and this may necessitate the delay of immunosuppression. Others, such as azole antifungals may need to be reinitiated immediately postLTx and significant modifications may be required. Table 3 gives an overview of significant interactions to consider in the peri-operative period.

Traditional antiviral treatments for HIV or hepatitis C contributed to complex drug-drug interactions with immunosuppressant regimens. Modern anti-retroviral and hepatitis therapies can now be safely administered post-LTx utilizing a standard immunosuppressant regimen without problematic interactions.

\section{Perioperative issues and immunosuppression}

LTx is commonly surgically challenging and unpredictable. A seemingly perfectly fine pre-LTx immunosuppression protocol may therefore need immediate modification in the early post-LTx period.

\section{Extensive intra-operative hemorrbage}

Effectively such patients will lose their pre-LTx induction immunosuppression with the replacement of lost blood volume. Almost universally an acute kidney injury (AKI) is associated, and in extreme cases hemofiltration via an extra-corporeal membrane oxygenation (ECMO) circuit is required. In the short term, corticosteroids, a reduced dose of the anti-proliferative agent and basiliximab are appropriate, while avoiding nephrotoxic agents such as CNI (2). However, if the AKI appears to be well established then implementing a reduced steroid dose and starting up the CNI may actually be a superior combination in providing anti-rejection cover while improving wound healing responses and decreasing the broad infection association of systemic steroids.

\section{Perioperative sepsis and septic shock}

Immunosuppression is required for rejection prophylaxis, but it needs to be recognized that in the setting of a serious septic event, anti-infective therapy and circulatory stabilization are the first priorities. Immunosuppression can be rationalized to a hydrocortisone infusion and IVIG as appropriate, or even a basiliximab 'holiday' (if not previously given) $(2,20)$. In the vast majority of cases the patient survives and the allograft will be relatively easily salvaged days to weeks subsequently.

\section{Altered conscious state and neurotoxicity}

Lung transplant recipients are particularly vulnerable to neurological complications post-surgery for several reasons (21). Chronic hypoxemia is common in those with end-stage lung diseases, hemodynamic instability from cardiopulmonary bypass devices and neurotoxicity from immunosuppression can all contribute to neurotoxicity (22). Encephalopathy is the most common neurological complication post- LTx with an incidence of $30 \%(15,21)$. Neurotoxicity from CNIs can manifest as headache, confusion or tremor to more serious consequences such as altered mental status, seizures or posterior reversible encephalopathy syndrome (PRES). Corticosteroids can exacerbate delirium in the peri-operative period and minimization of corticosteroid dose may also be necessary (15).

Patients with pre-existing neurological conditions leading into LTx who are at risk of complications are likely to benefit from delayed introduction of CNIs. Our 
Table 3 Specific recipient Drug Interactions used in potential LTx recipients that require modification of Maintenance Immunosuppression

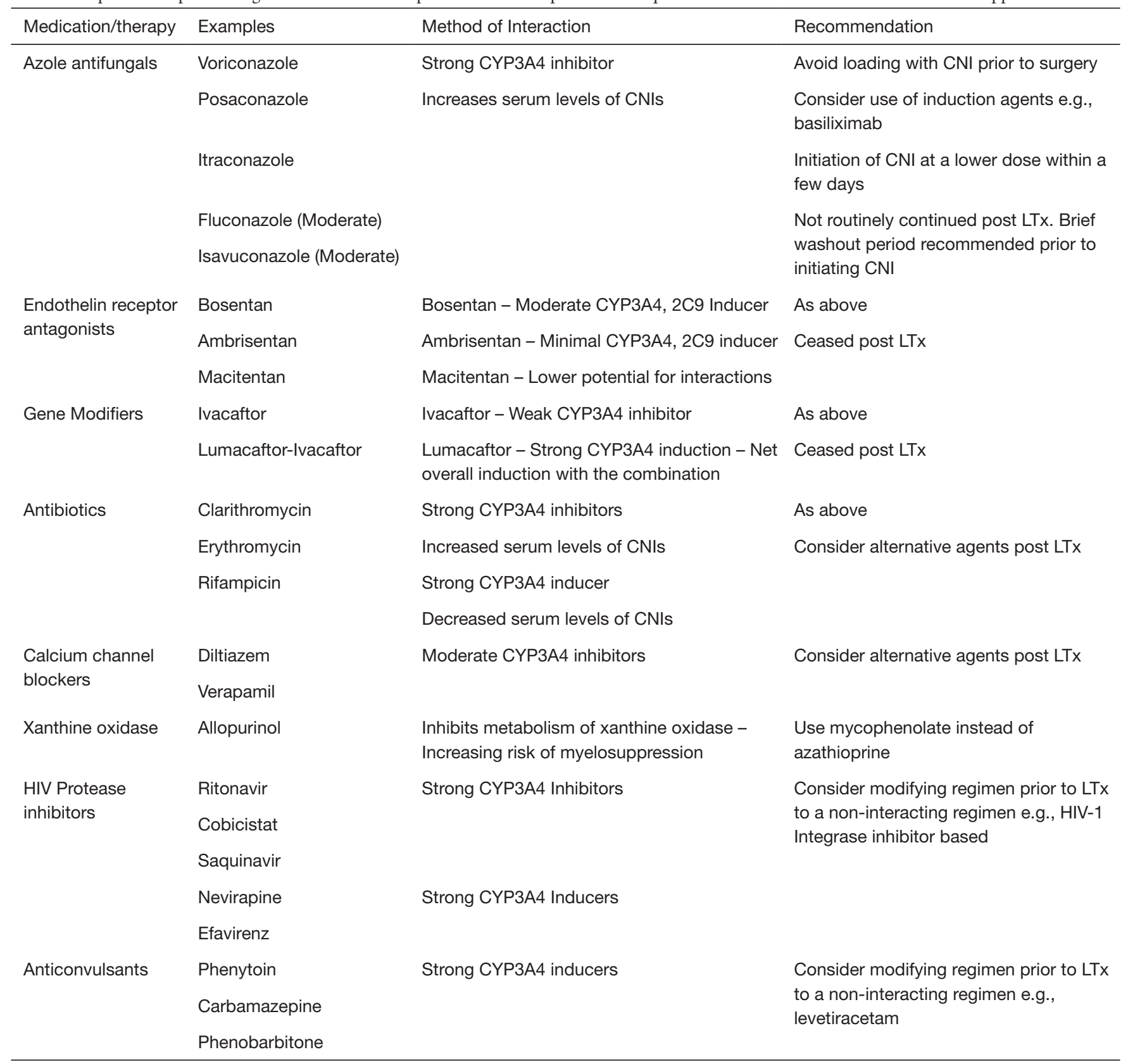

LTx, lung transplantation. CNI, calcineurin inhibitor.

approach to patients with an altered conscious state in the peri-operative period has been to withhold the CNI. The substitution of tacrolimus for cyclosporine may provide benefit in some patients.

Hyperammonemia is a rare but potentially fatal complication of SOT. Immunosuppressive agents and hepatic enzyme deficiency may play a role, but recently an infective component has been identified (23). The association with Ureaplasma infection has changed the approach to this syndrome. Measurement of ammonia levels is recommended in patients developing neurological symptoms (24).

\section{Absorption and gastrointestinal motility issues}

Tacrolimus based immunosuppressant regimes form the cornerstone of immunosuppression post LTx. Managing tacrolimus post LTx can be challenging due to a number 
of unique factors specific to LTx. Tacrolimus has a narrow therapeutic index, high intra-patient variability and TDM is essential for treatment individualization (25). Achieving therapeutic levels can be further complicated by a number of unique gastrointestinal factors specific to LTx. Patients with CF have unique pharmacokinetic profiles with delayed drug absorption as well as increased clearance of medications. Therefore, patients with CF require a higher $\mathrm{mg} / \mathrm{kg}$ dose of tacrolimus compared to those without CF (26).

The motility of the gastrointestinal tract may be significantly altered in the perioperative phase. The duodenum, the primary site of tacrolimus absorption may be significantly impacted in hemodynamically unstable patients. The oral route is the preferred route of administration, but sublingual or intravenous may be useful for a limited period of time. However, sublingual absorption of tacrolimus is minimal, whilst intravenous administration may be limited by lack of available lumens and higher rates of neuro and nephrotoxicity (27).

\section{Immunosuppressive drug metabolism and dosing on ECMO and dialysis}

The most common reason for modification of induction immunosuppression regimens is pre-existing renal impairment. Renal failure in the acute setting after LTx increases the risk of early and late post-LTx morbidity and mortality (28). Indeed, in one study (29) the incidence of AKI post-LTx was $52.5 \%$, with $9.3 \%$ of patients will requiring renal replacement therapy (RRT). Screening for high-risk patients at the time of LTx will minimize the risk of AKI as CNI minimization strategies can then be put in place. The use of induction agents such as basiliximab or alemtuzumab may delay the need for the early utilization of CNIs (30).

CNIs cause AKI through direct vasoconstriction of the afferent and efferent arterioles. High levels of unbound plasma concentrations of tacrolimus can contribute to toxicity, even when tacrolimus concentrations are in the therapeutic range. The potent vasoconstriction is likely caused by higher levels of endothelin and impaired production of nitric oxide (31).

In the LTx peri-operative phase, the pharmacokinetics of immunosuppressants can become markedly complex. Factors that may impact the pharmacokinetics of tacrolimus in the critically ill patient include inflammation, hypoalbuminemia, blood transfusions and hypotension.

ECMO can further impact drug pharmacokinetics by increasing the volume of distribution requiring more drug to achieve the same concentration as well as potential sequestration into the circuit (32). Additionally, absorption and sequestration of immunosuppressants (tacrolimus and mycophenolate) in the ECMO bypass circuit can be expected as they are highly lipophilic and protein bound drugs. However, at least in a heart transplant study, levels may rise and doses may need to be decreased (33).

\section{Modification of maintenance immunosuppression}

\section{Host factors identified at baseline}

Once the early post LTx period has passed and the acute challenges dealt with, a maintenance immunosuppression strategy must be considered. Immunosuppression is traditionally maintained with maintained with triple therapy consisting of a CNI (usually tacrolimus), anti-proliferative (mycophenolate or azathioprine) and corticosteroids. Dosing is adjusted over time to reflect the changes in immune tolerance to the allograft and is reduced in most cases over the first 12 months to a baseline prednisolone dose of 5-10 mg per day and a tacrolimus trough level target of 4-8 mcg/L (Table 4). There are various scenarios however which give rise to varying the standard regime.

Many transplant recipients have factors identified at baseline, which leads to alteration of the regime or target drug levels. These have been already been outlined above. Many of these modifications to the induction regime often persist for a long course post-LTx, if not indefinitely, reflecting the non-modifiable host factors that impact the net immunosuppression strategy in patients.

\section{Drug interactions}

The addition of interacting medications has the ability to destabilize immunosuppressant regimens. The most frequently encountered would be the addition of azole antifungals (posaconazole, voriconazole, itraconazole and fluconazole) for the treatment of Aspergillus. The extent of the interaction cannot always be predicted and despite preemptive dosage reduction when initiated, there is a large amount of inter-patient variability. Patients initiating or ceasing azoles are at greatest risk of variability.

The advent of novel oral anticoagulant (NOAC) agents for venous thromboembolism and atrial fibrillation has been variable for LTx recipients requiring anticoagulation. While there has been uptake of NOAC usage for LTx recipients, this can be problematic. While there are no significant changes in tacrolimus levels, the effects of NOACs have 
Table 4 The Alfred's maintenance immunosuppression dosing guidelines

\begin{tabular}{|c|c|c|c|c|}
\hline Months & $0-3$ & $3-6$ & $6-12$ & $>12$ \\
\hline Azathioprine & \multicolumn{4}{|c|}{$1.5 \mathrm{mg} / \mathrm{kg} / \mathrm{day}$} \\
\hline Mycophenolate & \multicolumn{4}{|c|}{$0.5-1 \mathrm{~g}$ twice daily } \\
\hline Prednisolone (mg/day, weight $>50$ kg) & 20 & 15 & 12.5 & $5-10$ \\
\hline Cyclosporine 2-hour (C2) level & & & $1,000-1,200$ & $400-800$ \\
\hline Sirolimus level & \multicolumn{4}{|c|}{$4-8$} \\
\hline Everolimus level & \multicolumn{4}{|c|}{$4-8$} \\
\hline
\end{tabular}

only been studied in kidney transplant recipients (34). Furthermore there is a contraindication to using NOAC agents in patients already on azole antifungals, highlighting the ongoing complex nature of medication management post-LTx (2).

\section{Allograft dysfunction}

Lung allograft rejection remains the main barrier to achieving better long-term outcomes post-LTx. Acute cellular rejection (ACR) on transbronchial biopsies signals acute allograft failure, but is a risk factor for the development of chronic lung allograft dysfunction (CLAD) (35). Tacrolimus level variability increases the risk of ACR and CLAD, particularly if occurring after the first 6 months post-LTx (2). The mainstay of management in this scenario is pulsed corticosteroids (intravenous methylprednisolone, 500-1,000 $\mathrm{mg}$ daily for three days) in addition to reviewing medication compliance and dosing. Additional factors contributing to tacrolimus variability are reviewed including absorption and drug interactions as well as aiming for a higher target tacrolimus trough level. Those on azathioprine can be transitioned to mycophenolate as the preferred anti-proliferative agent with some trial evidence of potential for a reduction in subsequent ACR, CLAD and improved graft survival (36).

Antibody-mediated rejection (AMR) remains a challenging entity without a definitive histopathological pattern, but rather relies on a constellation of clinical, histopathological and immunological features to formulate a diagnosis (37). This challenge also translates to treating AMR given the difficulties in diagnosis and also ongoing assessment. Nevertheless, when treatment of AMR is undertaken, there are acute and longer term management strategies for immunosuppression. After a pulse of corticosteroids, further management of AMR includes a combination of IVIG, plasmapheresis and a proteasome inhibitor (e.g., bortezomib) or anti-CD20 monoclonal antibody (e.g., rituximab) (38). Concurrently baseline immunosuppression is reviewed and changes may be instituted to enhance baseline immunosuppressant levels: including increasing the corticosteroid dose (often to $10 \mathrm{mg}$ per day or more), ensuring mycophenolate is the antiproliferative agent used (or at a higher dose- $750-1,000 \mathrm{mg}$ twice daily), as well as targeting a higher tacrolimus trough level.

Where CLAD is suspected or established, azithromycin is added to the regime to improve allograft function (39). More recently while there is emerging evidence that montelukast may have a role in established CLAD to reduce the rate of lung function decline and improve outcomes, this is not currently standard practise (40).

\section{Renal dysfunction}

Renal impairment remains a common and challenging issue post-LTx. AKI can be due to peri-operative issues and maybe short term as discussed above. AKI is a risk factor for chronic kidney disease (CKD), however, the predominant etiology for CKD are nephrotoxins, namely CNIs (41). CNI have both short (renal vasoconstriction) and longer term (chronic arteriolar changes and interstitial fibrosis) effects (28). While the severity of CKD amongst LTx recipients is variable, $5-10 \%$ of recipients have severe CKD (creatinine $>221 \mu \mathrm{mol} / \mathrm{L}$ or a need for dialysis or renal transplant) within 1 year post LTx, with $16 \%$ and $25 \%$ having CKD by 5 and 10 years respectively (3). 
Management of CKD post-LTx includes reducing target tacrolimus levels (42) or switching to mTORbased immunosuppression. Early transition to an mTOR inhibitor is not recommended due to the risk of delayed wound healing, but ideally LTx recipients should be switched prior to the onset of proteinuria so as to increase the likelihood of renal recovery (43). Management of other reno-vascular disease factors remains paramount, including aggressive management of hypertension and diabetes (both known complications of tacrolimus and steroid-based immunosuppression), to ensure all possible sources of renal injury are targeted appropriately.

\section{Gastrointestinal issues}

LTx immunosuppression plays a major role in the development of Gastrointestinal (GI) complications, due to the relatively high doses given. It can be difficult to differentiate whether the complication is due to infection due to the immunosuppressed nature or a direct action of the immunosuppression (44).

Nausea is the most common GI complaint post-LTx, attributed to side-effects of immunosuppressants such as mycophenolate, azathioprine or other commonly prescribed medications such as valganciclovir (44). Persistent nausea can be attributed to gastroparesis, an unfortunate surgical complication post-LTx can be caused by intra-operative vagal nerve damage or medications such as narcotic analgesics (45). Immunosuppressant levels may become subtherapeutic secondary to gastric stasis. As doses are escalated to achieve a pre-determined protocolized target level, there is potential for supra-therapeutic levels once gut motility is restored, with an abrupt rise to toxic concentrations. Although symptoms usually resolve within 6 months postLTx, there are concerns with the risk of aspiration from a poorly emptying stomach and the subsequent eventual development of CLAD (46).

CF may be associated with liver disease as well as motility-related issues due to mutations of the chloride channel resulting in gastroparesis, meconium ileus and more severe manifestations such as distal intestinal obstruction syndrome. Pancreatic insufficiency will impact the absorption of medications. Patients with CF have erratic gastrointestinal absorption impacting on achieving adequate levels potentially resulting in lower immunosuppression exposure (47).

Hepatotoxicity can also be problematic post-LTx. Many commonly prescribed medications post-LTx all contribute to hepatic dysfunction such as cell anti-proliferatives, azole antifungals and infrequently CNIs. Lung transplant recipients treated with voriconazole have a higher incidence of hepatotoxicity compared to other SOTs and the risk is greatest when initiated within 30 days post-LTx. Additional serious gastrointestinal complications that may occur including cholecystitis, intestinal perforation, pancreatitis and diverticulitis (48).

Situations with increased gastrointestinal motility, such as diarrhoeal illness can result in increased serum concentrations of immunosuppressants. CNIs and mTOR inhibitors are metabolized by CYP3A and p-glycoprotein in the gut wall and decreased transient times result in potential toxicity of tacrolimus. Patients with diarrhoeal illness are likely to have supra-therapeutic tacrolimus levels on admission with associated AKI. Our approach in this situation is to withhold the CNI or mTOR inhibitor until the AKI has resolved and reintroduce cautiously (49).

The incidence of gastro-esophageal reflux disease (GERD) is high post-LTx and has been linked to the development of CLAD (50). Steroid immunosuppression can contribute to the development of duodenal ulcers or poor wound healing (51). Dose reduction, temporary cessation, or discontinuation of certain immunosuppressive drugs is an important strategy to manage GI toxicities. However, the duration of these interventions can potentially increase the risk of graft rejection, if inadequate immunosuppression is not provided through routes or strategies.

\section{Metabolic issues}

Immunosuppressants can contribute to cardiovascular risk factors such as hypertension, hyperlipidemia, diabetes mellitus (DM) and renal disease. DM is common post-LTx and can be associated with immunosuppressant regimens containing corticosteroids or CNIs, while $50 \%$ of CF patients being considered for LTx having pre-existing DM $(52,53)$. Glucose intolerance is more likely in patients receiving tacrolimus than cyclosporine (25). The emergence of DM post-LTx is not an indication to switch CNIs or aim for lower therapeutic levels. Instead, close monitoring of glucose levels and the implementation of standard DM therapies is essential to prevent long-term morbidity associated with under-treated DM.

Hypertension, caused by renal vasoconstriction and sodium retention develops relatively early following CNI initiation and can lead to the development of CKD, stroke 
and other cardiovascular complications post-LTx (54). Calcium channel blockers are the agents of choice for CNI-induced hypertension, as they reverse CNIinduced vasoconstriction. Hyper-cholesterolemia or hyper-triglyceridemia are more common with CsA than Tacrolimus (53). CNIs, predominantly cyclosporine, have the potential to significantly increase statin exposure and those metabolized via cytochrome $\mathrm{P}_{450}$ enzymes (atorvastatin and simvastatin) are at higher risk of potential rhabdomyolysis (55).

Given the long term cardiovascular and renal consequences of uncontrolled hypertension, hypercholesterolemia and DM, management of these predictable side-effects is essential to minimize long-term morbidity.

\section{Hematological issues}

Leukopenia of varying severities can be attributed to several immunosuppressants utilized in standard LTx regimens. Azathioprine, and to a lesser extent mycophenolate, are the most likely agents to cause leukopenia. Leukopenia associated with azathioprine is usually reversible with dose reduction or discontinuation (18). Trough levels of the active metabolite of mycophenolate (mycophenolic acid $=$ MPA) is related to the risk of leukopenia associated with this drug. However, trough levels are not routinely performed at many institutions. Current practices include adjusting doses to maintain a white cell count of $>4,000$. Co-administration of the antimetabolites with other medications such as valganciclovir and co-trimoxazole are other frequently implicated as causative factors in the high incidence of leukopenia post-LTx (56).

Patients with mutations of the telomerase complex are at high risk of hematological complications following LTx. Thrombocytopenia is the most common hematological complication, followed by anemia and neutropenia. LTx recipients with this manifestation may have mycophenolate or azathioprine removed from the drug regimen after LTx without undue rejection consequences (57).

The incidence of anemia post-LTx has been reported at an incidence of $65 \%$ (31). The development of anemia is closely linked to post-LTx renal impairment. The use of erythropoietin has been administered to improve hemoglobin levels (31). The anti-metabolites (e.g., azathioprine and to a lesser extent mycophenolate) and mTOR inhibitors have all been implicated as causes of anemia. Although relatively rare, hemolytic anemia is not associated with immunosuppression, but more commonly with therapies for Pneumocystis Firovecii Pneumonia prophylaxis (e.g., dapsone or primaquine).

Thrombotic thrombocytopenic purpure (TTP) is an infrequent, but serious, complication attributed to CNIs. CNIs can injure endothelial cells, cause vasoconstriction and increase platelet aggregation resulting in TTP (58). Neurological symptoms or a decline in renal function may be attributed to CNI-related neurotoxicity or nephrotoxicity confounding a TTP diagnosis (59). TTP can occur at any time post LTx and is independent of levels, with the highest risk when an mTOR inhibitor is used in conjunction with a CNI (58).

\section{Advanced age and frailty strategies}

The number of elderly patients receiving LTx has increased significantly in recent years (60). The elderly are impacted by immunosenescence that is characterized by a gradual deterioration of the immune system with impairment of adaptive or innate immune response (61). Immunosenescence is likely to increase the risk of DM, bacterial infections and malignancies, although may be associated with lower rates of acute rejection. Agerelated changes can result in altered pharmacokinetics of immunosuppressants in the elderly resulting in increased medical comorbidities, including hypertension, renal dysfunction and hyperlipidemia (60-62). Despite these distinct changes, there are no specific recommendations for maintenance immunosuppression in the elderly. Clinicians may intuitively reduce the baseline immunosuppression in the elderly due to their susceptibility to side effects and infection. A reduced CNI target concentration and lower corticosteroid maintenance dose is appropriate with the aim of reducing the risk of nephrotoxicity and the risk of metabolic complications.

Frailty, generally defined as a functional decline with decreased reserve across multiple physiologic systems, is associated with poorer outcomes post-LTx (63). Symptoms of frailty may overlap with some of those seen in ageing, although it can also be seen in younger patients (64). There is a paucity of information regarding the correct approach to immunosuppression in the frail patient. However, frailty has a number of distinct characteristics including a chronic inflammatory, pro-coagulant and sarcopenic state that can all impact immunosuppressant pharmacokinetics (64). 


\section{Chronic infection issues}

Chronic and recurrent infections post-LTx remains a challenge and significant contributor to CLAD, morbidity and mortality. Bacterial infections remain problematic, particularly in those who come into LTx with suppurative lung disease such as CF and other types of bronchiectasis, particularly with sinus and proximal airway pathogen colonization. In these patients, an active reduction in maintenance immunosuppression is appropriate, often via cessation of the anti-proliferative agent, potentially with the addition of IVIG.

This same strategy is applied in those with recurrent CMV infection. Epstein-Barr virus (EBV) reactivation remains a less common occurrence post LTx. EBV is predominantly seen in those who are an EBV mismatch (donor positive, recipient negative) and should be considered for life-long prophylactic antiviral therapy. The development of EBV-driven post-transplant lymphoproliferative disease (PTLD) is problematic in a significant portion of the mismatched LTx recipients, likely associated with the greater volume of lymphoid tissue in the transplanted lung (compared to other SOT) (65). This requires more aggressive reduction in immunosuppression with cessation of the anti-proliferative agent and a reduction in tacrolimus target levels to the lowest acceptable levels.

LTx for patients with concurrent blood borne viruses (BBV), namely hepatitis B (HBV), hepatitis C (HCV) and human immunodeficiency virus (HIV), is currently a rare occurrence. Similarly, the transmission of donor-acquired $\mathrm{BBV}$ via transplantation remains rare, even in those who are deemed high risk donors (66).

Historically, a BBV was a contraindication to SOT, but with the advent of newer therapies with more favorable side-effect profiles has the real prospect of viral cure in those with HCV and good disease control in those with HBV and HIV, LTx is now a therapeutic option in these patients. HBV treatment is limited to interferon-free regimes, due to the risk of allograft rejection with interferon therapy (67). HCV treatment has been revolutionized with direct-acting antiviral agents to a degree that $\mathrm{HCV}$ infected donors are now knowingly utilized and their organs transplanted to $\mathrm{HCV}$ negative recipients, expanding the donor pool significantly. These HCV therapies do not interact with conventional immunosuppression, are only required for a short duration if commenced early postLTx and have demonstrated excellent virological response rates. Subsequent lung allograft function and survival are equivalent to those seen with conventional non-HCV donors (68).

While liver and renal transplantation has become standard practice for those patients with HIV and endorgan disease, LTx in this population remains relatively recent and limited (11). Evidence to date suggests increased rates of ACR but similar survival in LTx recipients with HIV compared to the standard cohort, which is in keeping with the outcomes seen in other SOT. This may in part be due to drug interactions between CNI and HIV antiretroviral agents (ARV), namely protease inhibitors or nonnucleoside reverse transcriptase inhibitors, which affects CNI metabolism via cytochrome $\mathrm{P} 450-3 \mathrm{~A}$ (11). This issue has been successfully managed by switching patients with HIV from these interacting agents to an integrase inhibitor based ARV regime and ensuring stability of HIV control prior to LTx. This allows conventional immunosuppression to be commenced in these patients post-LTx without concern for complex drug interactions (2).

\section{Malignancy strategies}

The risk of malignancy post LTx is predominantly due to immunosuppression with a 2.5 - to 3 -fold increased risk compared to the general population (65). Skin cancers, in particular, are common post LTx. In the setting of recurrent non-melanoma skin cancers or any other malignancy, immunosuppression is aggressively reduced with cessation of the anti-proliferative agent. Tacrolimus target levels are reduced or LTx recipients are switched to an mTOR inhibitor given its potential to reduce the risk of malignancy via its action on anti-proliferation and cell metabolism (69).

\section{Future strategies and direction of LTx immunosuppression}

\section{Nebulized LTx immunosuppression}

The theoretical advantage of nebulized immunosuppression is that additional doses of conventional immunosuppression may be administered while encountering less systemic side effects such as renal or hepatic impairment (70-73). An initial randomized trial found that inhaled cyclosporine did not improve the rate of ACR, but did improve survival and periods of chronic rejection-free survival (74). However, a larger multi-center, randomized did not improve CLADfree survival or overall mortality (75). A small study of inhaled tacrolimus has additionally been undertaken (76). 


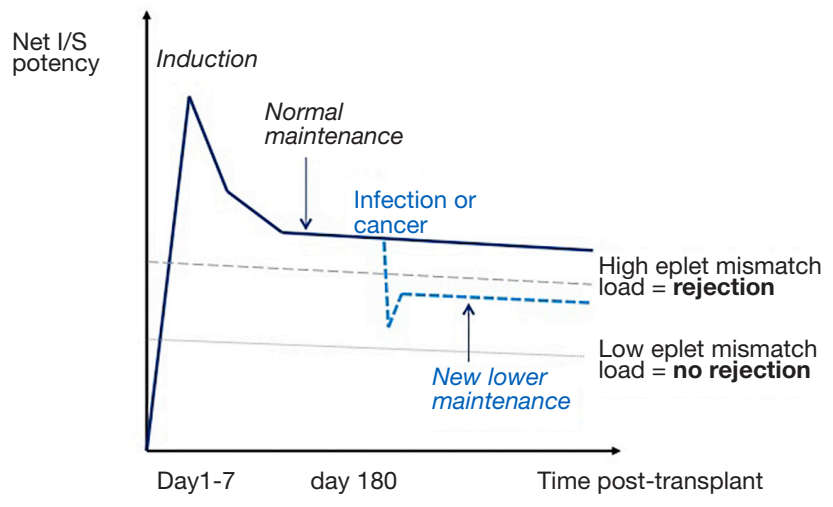

Figure 3 The potential of allo-immunity risk profiling versus immunosuppression.

A randomized trial of inhaled corticosteroids did not show significant benefit (77).

\section{Allo-immunity risk profiling to understand absolute allograft risk}

Wiebe and colleagues have recently shown that is possible to use a calculated score (HLA Matchmaker, Pittsburgh, PA, USA) of the extent of donor-recipient immunological mismatch to stratify the net requirement for post-renal transplant immunosuppression (78). Figure 3 gives a schematic view of how this measure could be utilized clinically.

\section{Novel markers to quantify the impact of immunosuppressive medication}

Best characterized following liver transplantation, the ImmuKnow (Cylex, Columbia, MD, USA) assay is a potential tool to determine the net overall state of immunosuppression post-transplant (79). Bhorade and colleagues found that ImmunoKnow levels were lower in infected LTx recipients compared with non-infected recipients (80).

The QuantifERON Monitor (QFM)(Qiagen, Hombrechtikon, Switzerland) provides assessment of an individual's cell-mediated response through dual innate (R848) and adaptive (anti-CD3) immune system stimulation, providing both qualitative and quantitative analysis of cell-mediated immunity. Studies in other SOT $(81,82)$ populations have shown the QFM is able to quantify the net level of immunosuppression and predict the risk of subsequent infection episodes.

Other biomarkers may also potentially reflect the total degree of immunosuppression $(83,84)$. Torque Teno virus (TTV) is a human DNA virus that causes persistent asymptomatic viremia in the general population. Replication of TTV is subject to immune control (85). Recently, it was found an association between high plasma TTV levels and the development of CLAD, suggesting that TTV monitoring may be useful in identifying those at highest risk (86).

Measuring the biological activity of immunosuppressants on intracellular target enzymes is an alternate approach to measuring whole blood levels. Measuring inosine monophosphate dehydrogenase levels correlates with mycophenolic acid activity (87) and measuring intracellular concentrations of CNIs correlates more tightly than blood levels with efficacy (88) and infection risk (89).

\section{Detecting sub-clinical allograft dysfunction from any cause}

Allograft function is historically assessed by the measurement of lung function, however early sub-clinical alloimmune activation may well go undetected in the absence of overt spirometric change. The detection of graftderived cell free DNA in the blood is a recognized marker of early allograft dysfunction $(90,91)$. It is a non-invasive measure that is specific for damage to the transplanted allograft. In the LTx setting it is also possible to measure directly from the allograft by utilizing bronchoalveolar lavage samples.

A number of other biomarkers that may also predict early graft changes have been described (92). These assessments are providing insights into the mechanisms of CLAD development and may identify potential immunosuppressive targets prior to the development of clinically evident disease. Notwithstanding, there are real challenges translating a promising research tool into a routine clinical practice measure (93).

\section{Molecular assessment of rejection and injury}

Extending work done in renal and cardiac transplantation, a microarray-based diagnostic system (Molecular Microscope ${ }^{\circledR}$ Diagnostic System, Edmonton, Canada) has recently emerged as an alternative to conventional histology (94). The system features a microarray-based central diagnostic system developed for real time assessment by defining rejection-associated transcripts that consist of 200 probe sets 
associated with acute rejection (both T cell- and antibodymediated), non-specific lung injury (infection) and normal allograft function (94). These analyses can be performed on transbronchial and endobronchial biopsies $(94,95)$, as well as blood (96) and BAL (97).

Based on renal transplant work (98), it is now possible to go one step deeper and assess lung cell transcriptomics using single-cell RNA-sequencing (scRNAseq).

\section{Conclusions}

With increasingly complex LTx candidates being successfully transplanted and improved survival in these patients, immunosuppression regimes have evolved from a standard recipe to fit all types into a nuanced and tailored individualized approach to balance the risk of immunosuppression against the competing risk of rejection. This requires careful consideration of a single recipient's unique multiple inherent host factors, their perioperative course and immunosuppressant side-effects, all the while targeting the minimization of comorbidities and CLAD while aiming for overall excellent long-term LTx outcomes. As our understanding of the many complex factors involved in allograft dysfunction expands, coupled with novel emerging molecular and biomarker assessments, immunosuppression strategies will continue to require ongoing evolution and personalization.

\section{Acknowledgments}

We acknowledge the support from the Lungitude Foundation.

Funding: None.

\section{Footnote}

Provenance and Peer Review: This article was commissioned by the Guest Editor (Jonathan D'Cunha) for the series "Lung Transplantation: Past, Present, and Future" published in Fournal of Thoracic Disease. The article has undergone external peer review.

Conflicts of Interest: All authors have completed the ICMJE uniform disclosure form (available at http:// dx.doi.org/10.21037/jtd-2021-11). The series "Lung Transplantation: Past, Present, and Future" was commissioned by the editorial office without any funding or sponsorship.
The authors have no other conflicts of interest to declare. Ethical Statement: The authors are accountable for all aspects of the work in ensuring that questions related to the accuracy or integrity of any part of the work are appropriately investigated and resolved.

Open Access Statement: This is an Open Access article distributed in accordance with the Creative Commons Attribution-NonCommercial-NoDerivs 4.0 International License (CC BY-NC-ND 4.0), which permits the noncommercial replication and distribution of the article with the strict proviso that no changes or edits are made and the original work is properly cited (including links to both the formal publication through the relevant DOI and the license). See: https://creativecommons.org/licenses/by-nc-nd/4.0/.

\section{References}

1. Paraskeva MA, Levin KC, Westall GP, et al. Lung transplantation in Australia, 1986-2018: more than 30 years in the making. Med J Aust 2018;208:445-50.

2. Ivulich S, Westall G, Dooley M, et al. The Evolution of Lung Transplant Immunosuppression. Drugs 2018;78:965-82.

3. Chambers DC, Cherikh WS, Harhay MO, et al. The International Thoracic Organ Transplant Registry of the International Society for Heart and Lung Transplantation: Thirty-sixth adult lung and heart-lung transplantation Report-2019; Focus theme: Donor and recipient size match. J Heart Lung Transplant 2019;38:1042-55.

4. Valapour M, Lehr CJ, Skeans MA, et al. OPTN/SRTR 2018 Annual Data Report: Lung. Am J Transplant 2020;20 Suppl s1:427-508.

5. Birch J, Sunny SS, Hester KLM, et al. Outcomes of lung transplantation in adults with bronchiectasis. BMC Pulm Med 2018;18:82.

6. Verma N, Grimbacher B, Hurst JR. Lung disease in primary antibody deficiency. Lancet Respir Med 2015;3:651-60.

7. Burton C, Milman N, Andersen C, et al. Common variable immune deficiency and lung transplantation. Scand J Infect Dis 2007;39:362-7.

8. Farmer JR, Sokol CL, Bonilla FA, et al. Bilateral lung transplantation in a patient with humoral immune deficiency: a case report with review of the literature. Case Reports Immunol 2014;2014:910215.

9. Mawhorter S, Yamani MH. Hypogammaglobulinemia and infection risk in solid organ transplant recipients. Curr 
Opin Organ Transplant 2008;13:581-5.

10. Heinbokel T, Elkhal A, Liu G, et al. Immunosenescence and organ transplantation. Transplant Rev (Orlando) 2013;27:65-75.

11. Koval CE, Farr M, Krisl J, et al. Heart or lung transplant outcomes in HIV-infected recipients. J Heart Lung Transplant 2019;38:1296-305.

12. Stephenson AL, Sykes J, Berthiaume Y, et al. Clinical and demographic factors associated with post-lung transplantation survival in individuals with cystic fibrosis. J Heart Lung Transplant 2015;34:1139-45.

13. Smibert O, Snell GI, Bills H, et al. Mycobacterium abscessus Complex - a Particular Challenge in the Setting of Lung Transplantation. Expert Rev Anti Infect Ther 2016;14:325-33.

14. Robinson CA, Inci I, Naegeli M, et al. Extracorporeal photopheresis as second-line treatment therapy in lifethreatening primary graft dysfunction following lung transplantation. Pediatr Transplant 2018;22:e13145.

15. Pizzi M, Ng L. Neurologic Complications of Solid Organ Transplantation. Neurol Clin 2017;35:809-23.

16. Kliman DS, Kotecha SR, Abelson DC, et al. Favorable Outcome of Lung Transplantation for Severe Pulmonary Graft Versus Host Disease: An Australian Multicenter Case Series. Transplantation 2019;103:2602-7.

17. Sugimoto S, Miyoshi K, Kurosaki T, et al. Favorable survival in lung transplant recipients on preoperative lowdose, as compared to high-dose corticosteroids, after hematopoietic stem cell transplantation. Int J Hematol 2018;107:696-702.

18. Danesi R, Del Tacca M. Hematologic toxicity of immunosuppressive treatment. Transplant Proc 2004;36:703-4.

19. Girnita DM, Burckart G, Zeevi A. Effect of cytokine and pharmacogenomic genetic polymorphisms in transplantation. Curr Opin Immunol 2008;20:614-25.

20. Swarup R, Allenspach LL, Nemeh HW, et al. Timing of basiliximab induction and development of acute rejection in lung transplant patients. J Heart Lung Transplant 2011;30:1228-35.

21. Mateen FJ, Dierkhising RA, Rabinstein AA, et al. Neurological complications following adult lung transplantation. Am J Transplant 2010;10:908-14.

22. Shigemura N, Sclabassi RJ, Bhama JK, et al. Early major neurologic complications after lung transplantation: incidence, risk factors, and outcome. Transplantation 2013;95:866-71.

23. Anwar S, Gupta D, Ashraf MA, et al. Symptomatic hyperammonemia after lung transplantation: lessons learnt. Hemodial Int 2014;18:185-91.

24. Wang X, Greenwood-Quaintance KE, Karau MJ, et al. Ureaplasma parvum causes hyperammonemia in a pharmacologically immunocompromised murine model. Eur J Clin Microbiol Infect Dis 2017;36:517-22.

25. Monchaud C, Marquet P. Pharmacokinetic optimization of immunosuppressive therapy in thoracic transplantation: part I. Clin Pharmacokinet 2009;48:419-62.

26. de Groot R, Smith AL. Antibiotic pharmacokinetics in cystic fibrosis. Differences and clinical significance. Clin Pharmacokinet 1987;13:228-53.

27. Snell GI, Ivulich S, Mitchell L, et al. Evolution to twice daily bolus intravenous tacrolimus: optimizing efficacy and safety of calcineurin inhibitor delivery early post lung transplant. Ann Transplant 2013;18:399-407.

28. Puttarajappa CM, Bernardo JF, Kellum JA. Renal Complications Following Lung Transplantation and Heart Transplantation. Crit Care Clin 2019;35:61-73.

29. Lertjitbanjong P, Thongprayoon C, Cheungpasitporn W, et al. Acute Kidney Injury after Lung Transplantation: A Systematic Review and Meta-Analysis. J Clin Med 2019;8:1713.

30. Sikma MA, van Maarseveen EM, van de Graaf EA, et al. Pharmacokinetics and Toxicity of Tacrolimus Early After Heart and Lung Transplantation. Am J Transplant 2015;15:2301-13.

31. Bloom RD, Doyle AM. Kidney Disease after Heart and Lung Transplantation. Am J Transplant 2006;6:671-9.

32. Dzierba AL, Abrams D, Brodie D. Medicating patients during extracorporeal membrane oxygenation: the evidence is building. Crit Care 2017;21:66.

33. Breslin N, Salerno D, Jandovitz N, et al. Tacrolimus dosing requirements are reduced in heart transplant recipients with severe primary graft dysfunction on ecmo support. J Heart Lung Transplant 2017;36:S151-2.

34. Bukhari MA, Al-Theaby A, Tawhari M, et al. Efficacy and safety of non-vitamin $\mathrm{K}$ antagonist oral anticoagulants post-kidney transplantation. World J Transplant 2019;9:134-44.

35. Roden AC, Aisner DL, Allen TC, et al. Diagnosis of Acute Cellular Rejection and Antibody-Mediated Rejection on Lung Transplant Biopsies: A Perspective From Members of the Pulmonary Pathology Society. Arch Pathol Lab Med 2017;141:437-44.

36. Speich R, Schneider S, Hofer M, et al. Mycophenolate mofetil reduces alveolar inflammation, acute rejection and graft loss due to bronchiolitis obliterans syndrome 
after lung transplantation. Pulmonary Pharmacology and Therapeutics 2010;23:445-9.

37. Levine DJ, Glanville AR, Aboyoun C, et al. Antibodymediated rejection of the lung: A consensus report of the International Society for Heart and Lung Transplantation. J Heart Lung Transplant 2016;35:397-406.

38. Hulbert AL, Pavlisko EN, Palmer SM. Current challenges and opportunities in the management of antibodymediated rejection in lung transplantation. Curr Opin Organ Transplant 2018;23:308-15.

39. Corris PA, Ryan VA, Small T, et al. A randomised controlled trial of azithromycin therapy in bronchiolitis obliterans syndrome (BOS) post lung transplantation. Thorax 2015;70:442-50.

40. Vos R, Eynde RV, Ruttens D, et al. Montelukast in chronic lung allograft dysfunction after lung transplantation. J Heart Lung Transplant 2019;38:516-27.

41. Sikma MA, Hunault CC, van de Graaf EA, et al. High tacrolimus blood concentrations early after lung transplantation and the risk of kidney injury. Eur J Clin Pharmacol 2017;73:573-80.

42. Robinson PD, Shroff RC, Spencer H. Renal complications following lung and heart-lung transplantation. Pediatr Nephrol 2013;28:375-86.

43. Letavernier E, Legendre C. mToR inhibitors-induced proteinuria: mechanisms, significance, and management. Transplant Rev (Orlando) 2008;22:125-30.

44. Bravo C, Gispert P, Borro JM, et al. Prevalence and management of gastrointestinal complications in lung transplant patients: MITOS study group. Transplant Proc 2007;39:2409-12.

45. Raviv Y, D'Ovidio F, Pierre A, et al. Prevalence of gastroparesis before and after lung transplantation and its association with lung allograft outcomes. Clin Transplant 2012;26:133-42.

46. Jamie Dy F, Freiberger D, Liu E, et al. Impact of gastroesophageal reflux and delayed gastric emptying on pediatric lung transplant outcomes. J Heart Lung Transplant 2017;36:854-61.

47. Gilljam M, Chaparro C, Tullis E, et al. GI complications after lung transplantation in patients with cystic fibrosis. Chest 2003;123:37-41.

48. Grass F, Schafer M, Cristaudi A, et al. Incidence and Risk Factors of Abdominal Complications After Lung Transplantation. World J Surg 2015;39:2274-81.

49. Mittal N, Thompson JF, Kato T, et al. Tacrolimus and diarrhea: pathogenesis of altered metabolism. Pediatr Transplant 2001;5:75-9.
50. Hadjiliadis D, Davis RD, Steele MP, et al. Gastroesophageal reflux disease in lung transplant recipients. Clin Transplant 2003;17:363-8.

51. Paul S, Escareno CE, Clancy K, et al. Gastrointestinal complications after lung transplantation. J Heart Lung Transplant 2009;28:475-9.

52. Hackman KL, Bailey MJ, Snell GI, et al. Diabetes is a major risk factor for mortality after lung transplantation. Am J Transplant 2014;14:438-45.

53. Silverborn M, Jeppsson A, Martensson G, et al. New-onset cardiovascular risk factors in lung transplant recipients. J Heart Lung Transplant 2005;24:1536-43.

54. Parekh K, Trulock E, Patterson GA. Use of cyclosporine in lung transplantation. Transplant Proc 2004;36:318s-22s.

55. Bellosta S, Corsini A. Statin drug interactions and related adverse reactions. Expert Opin Drug Saf 2012;11:933-46.

56. Dobrolet NC, Webber SA, Blatt J, et al. Hematologic abnormalities in children and young adults receiving tacrolimus-based immunosuppression following cardiothoracic transplantation. Pediatr Transplant 2001;5:125-31.

57. Borie R, Kannengiesser C, Hirschi S, et al. Severe hematologic complications after lung transplantation in patients with telomerase complex mutations. J Heart Lung Transplant 2015;34:538-46.

58. Hachem RR, Yusen RD, Chakinala MM, et al. Thrombotic microangiopathy after lung transplantation. Transplantation 2006;81:57-63.

59. Go O, Naqvi A, Tan A, et al. The spectrum of thrombotic thrombocytopenic purpura: a clinicopathologic demonstration of tacrolimus-induced thrombotic thrombocytopenic purpura in a lung transplant patient. South Med J 2008;101:744-7.

60. Yusen RD, Christie JD, Edwards LB, et al. The Registry of the International Society for Heart and Lung Transplantation: Thirtieth Adult Lung and Heart-Lung Transplant Report--2013; focus theme: age. J Heart Lung Transplant 2013;32:965-78.

61. Krenzien F, ElKhal A, Quante M, et al. A rationale for age-adapted immunosuppression in organ transplantation. Transplantation 2015;99:2258-68.

62. Vadnerkar A, Toyoda Y, Crespo M, et al. Age-specific complications among lung transplant recipients 60 years and older. J Heart Lung Transplant 2011;30:273-81.

63. Wilson ME, Vakil AP, Kandel P, et al. Pretransplant frailty is associated with decreased survival after lung transplantation. J Heart Lung Transplant 2016;35:173-8.

64. Exterkate L, Slegtenhorst BR, Kelm M, et al. Frailty and 
Transplantation. Transplantation 2016;100:727-33.

65. Na R, Grulich AE, Meagher NS, et al. Comparison of de novo cancer incidence in Australian liver, heart and lung transplant recipients. Am J Transplant 2013;13:174-83.

66. Kotecha S, Williams TJ. Extending the criteria for acceptable organ donors: balancing the risks. Med J Aust 2019;211:402-3.

67. Vallet-Pichard A, Fontaine H, Mallet V, et al. VIRAL hepatitis in solid organ transplantation other than liver. J Hepatol 2011;55:474-82.

68. Cypel M, Woolley A. Commentary: Use of hepatitis C virus viremic donors should be the standard of care. $\mathrm{J}$ Thorac Cardiovasc Surg 2020;159:2126-7.

69. Fine NM, Kushwaha SS. Recent Advances in Mammalian Target of Rapamycin Inhibitor Use in Heart and Lung Transplantation. Transplantation 2016;100:2558-68.

70. Corcoran TE, Niven R, Verret W, et al. Lung deposition and pharmacokinetics of nebulized cyclosporine in lung transplant patients. J Aerosol Med Pulm Drug Deliv 2014;27:178-84.

71. Corcoran TE, Smaldone GC, Dauber JH, et al. Preservation of post-transplant lung function with aerosol cyclosporin. Eur Respir J 2004;23:378-83.

72. Burkart GJ, Smaldone GC, Eldon MA, et al. Lung deposition and pharmacokinetics of cyclosporine after aerosolization in lung transplant patients. Pharm Res 2003;20:252-6.

73. Behr J, Zimmermann G, Baumgartner R, et al. Lung deposition of a liposomal cyclosporine A inhalation solution in patients after lung transplantation. J Aerosol Med Pulm Drug Deliv 2009;22:121-30.

74. Iacono AT, Johnson BA, Grgurich WF, et al. A Randomized Trial of Inhaled Cyclosporine in LungTransplant Recipients. N Engl J Med 2006;354:141-50.

75. Johnson BA, Zamora MR, Budev MM, et al. 172 Cyclosporine Inhalation Solution Does Not Improve Bronchiolitis Obliterans Syndrome-Free Survival Following Lung Transplant: Results from the CYCLIST Trial. J Heart Lung Transplant 2012;31:S66.

76. Hayes D Jr, Zwischenberger JB, Mansour HM. Aerosolized tacrolimus: a case report in a lung transplant recipient. Transplant Proc 2010;42:3876-9.

77. Whitford H, Walters EH, Levvey B, et al. Addition of inhaled corticosteroids to systemic immunosuppression after lung transplantation: a double-blind, placebocontrolled trial. Transplantation 2002;73:1793-9.

78. Wiebe C, Kosmoliaptsis V, Pochinco D, et al. HLA-DR/ DQ molecular mismatch: A prognostic biomarker for primary alloimmunity. Am J Transplant 2019;19:1708-19.

79. Rodrigo E, Lopez-Hoyos M, Corral M, et al. ImmuKnow as a diagnostic tool for predicting infection and acute rejection in adult liver transplant recipients: a systematic review and meta-analysis. Liver Transpl 2012;18:1245-53.

80. Bhorade SM, Janata K, Vigneswaran WT, et al. Cylex ImmuKnow assay levels are lower in lung transplant recipients with infection. J Heart Lung Transplant 2008;27:990-4.

81. Sood S, Yu L, Visvanathan K, et al. Immune function biomarker QuantiFERON-monitor is associated with infection risk in cirrhotic patients. World J Hepatol 2016;8:1569-75.

82. Mian M, Natori Y, Ferreira V, et al. Evaluation of a Novel Global Immunity Assay to Predict Infection in Organ Transplant Recipients. Clin Infect Dis 2018;66:1392-7.

83. Gieser G, Harigaya H, Colangelo PM, et al. Biomarkers in solid organ transplantation. Clin Pharmacol Ther 2011;90:217-20.

84. Roedder S, Vitalone M, Khatri P, et al. Biomarkers in solid organ transplantation: establishing personalized transplantation medicine. Genome Med 2011;3:37.

85. Gorzer I, Haloschan M, Jaksch P, et al. Plasma DNA levels of Torque teno virus and immunosuppression after lung transplantation. J Heart Lung Transplant 2014;33:320-3.

86. Gorzer I, Jaksch P, Strassl R, et al. Association between plasma Torque teno virus level and chronic lung allograft dysfunction after lung transplantation. J Heart Lung Transplant 2017;36:366-8.

87. van Gelder T. Biomarkers in solid organ transplantation. Br J Clin Pharmacol 2017;83:2602-4.

88. Capron A, Haufroid V, Wallemacq P. Intra-cellular immunosuppressive drugs monitoring: A step forward towards better therapeutic efficacy after organ transplantation? Pharmacol Res 2016;111:610-8.

89. Greenland JR, Chong T, Wang AS, et al. Suppressed calcineurin-dependent gene expression identifies lung allograft recipients at increased risk of infection. Am J Transplant 2018;18:2043-9.

90. Agbor-Enoh S, Jackson AM, Tunc I, et al. Late manifestation of alloantibody-associated injury and clinical pulmonary antibody-mediated rejection: Evidence from cell-free DNA analysis. J Heart Lung Transplant 2018;37:925-32.

91. Whitlam JB, Ling L, Skene A, et al. Diagnostic application of kidney allograft-derived absolute cell-free DNA levels during transplant dysfunction. Am J Transplant 2019;19:1037-49. 
92. Tissot A, Danger R, Claustre J, et al. Early Identification of Chronic Lung Allograft Dysfunction: The Need of Biomarkers. Front Immunol 2019;10:1681.

93. Naesens M, Friedewald J, Mas V, et al. A practical guide to the clinical implementation of biomarkers for subclinical rejection following kidney transplantation. Transplantation 2020;104:700-7.

94. Halloran KM, Parkes MD, Chang J, et al. Molecular assessment of rejection and injury in lung transplant biopsies. J Heart Lung Transplant 2019;38:504-13.

95. Halloran K, Parkes MD, Timofte IL, et al. Molecular phenotyping of rejection-related changes in mucosal

Cite this article as: Kotecha S, Ivulich S, Snell G. Review: immunosuppression for the lung transplant patient. J Thorac Dis 2021;13(11):6628-6644. doi: 10.21037/jtd-2021-11 biopsies from lung transplants. Am J Transplant 2020;20:954-66.

96. Danger R, Royer PJ, Reboulleau D, et al. Blood Gene Expression Predicts Bronchiolitis Obliterans Syndrome. Front Immunol 2017;8:1841.

97. Weigt SS, Wang X, Palchevskiy V, et al. Gene Expression Profiling of Bronchoalveolar Lavage Cells Preceding a Clinical Diagnosis of Chronic Lung Allograft Dysfunction. Ann Am Thorac Soc 2017;14:S252.

98. Zhang W, Yi Z, Wei C, et al. Pretransplant transcriptomic signature in peripheral blood predicts early acute rejection. JCI Insight 2019; 4:e127543. 